\title{
Magnetic Behavior at Low Temperature of Carbon Foams Prepared by the Controlled Pyrolysis of Saccharose
}

 \\ ${ }^{a}$ Chemistry Centre, Venezuelan Institute for Scientific Research, 21827, Caracas 1020-A, Venezuela \\ ${ }^{b}$ Carlson School of Chemistry and Biochemistry, Clark University, Worcester, MA 01610, USA \\ ${ }^{c}$ Department of Physics, Clark University, Worcester, MA 01610, USA \\ ${ }^{d}$ Physics Centre, Venezuelan Institute for Scientific Research, 21827, Caracas 1020-A, Venezuela
}

\begin{abstract}
Carbon films and foams were obtained by the controlled pyrolysis of saccharose. Their irregular topology was confirmed by SEM. The presence of unpaired electrons was established via EPR spectroscopy. Magnetization as a function of both temperature and applied external field was collected using a SQUID Magnetometer and it was confirmed that the samples were paramagnetic at low temperatures (i.e $1.8 \mathrm{~K}-10 \mathrm{~K}$ ) and diamagnetic at temperatures higher than $10 \mathrm{~K}$. Results showed that saccharose can be used as a precursor for the synthesis of carbon materials for magnetic applications at low temperature.
\end{abstract}

Magnetizm is a property conventionally associated with transition metals and transition metal complexes with unpaired electrons. In one allotropic form, carbon exists as graphite, which is structurally highly regular and contains no unpaired electrons. However, some structurally highly irregular carbon materials, with high surface areas have been reported to display magnetic properties [1]. Magnetic properties of carbon materials depend on its allotropic modification, which is important because these solid carbon samples may potentially be employed as optoelectronic and photovoltaic devices [2]. Particularly, in the case of graphite or activated carbon, the magnetic properties are governed by circular currents driven on and between graphene layers. Recent studies have shown the influence of defects and dislocations in graphene layers in carbon nanofoams [3] and physisorption in microporous carbon [4] on magnetic behavior of carbon materials. The main objective of this work is to verify the magnetic behavior of carbon foams. Samples were synthesized by the controlled pyrolysis of saccharose previously dissolved at $80^{\circ} \mathrm{C}$ in water [5] or in $\mathrm{KOH}$ aqueous solution [6], denoted $A_{N W-1}$ and $A_{N W-2}$, respectively. Samples were recrystallized at $25^{\circ} \mathrm{C}$, thermally stabilized under vacuum (200mbar) at $110^{\circ} \mathrm{C}$ by $1 \mathrm{~h}$ and carbonized by $1 \mathrm{~h}$ under $\mathrm{N}_{2}$ flow at $450^{\circ} \mathrm{C}$. Carbon films and foams were obtained simultaneously and the highly irregular topology of samples was confirmed by Scanning Electron Microscopy (SEM). The presence of unpaired electrons in $\mathrm{A}_{\mathrm{NW}-1}$ and $\mathrm{A}_{\mathrm{NW}-2}$ was established by Electron Paramagnetic Resonance (EPR) spectra at ambient temperature in vacuumed-sealed quartz samples in an X-Band EMX Bruker Spectrometer. Magnetization as a function of temperature and applied external field was

*Address correspondence to this author at the Chemistry Centre, Venezuelan Institute for Scientific Research, 21827, Caracas 1020-A, Venezuela; Fax: +58-212-5041922; E-mail: jmatos@ivic.ve collected using a Quantum Design MPMS-XL SQUID Magnetometer.

Fig. (1) shows the SEM image of $A_{N W-1}$. The random topological structure of the carbon foam and the lack of structural regularity can be seen. This random organization of carbon foams in the shape of polygonal cells with n-sides have been reported previously [5,6]. It is also important to note the presence of a light hyperbolic curvature in the surface of this carbon foam. This could be associated to the presence of topological and bonding defects in graphene layers as suggested by Rode and co-workers [3]; however, to confirm this, it would be necessary to take high-resolution transmission electron microscopies. The presence of free electrons or radicals is readily observable in both $\mathrm{A}_{\mathrm{NW}-1}$ and $A_{N W-2}$ by simple observation of the respective EPR spectra in Fig. (2). Furthermore, the measured Lande factor or $g$ factor is 2.0075 for $\mathrm{A}_{\mathrm{NW}-1}$ and 2.0052 for $\mathrm{A}_{\mathrm{NW}-2}$, very close to that of a free electron $(\mathrm{ge}=2.002319)$. The line width is also very similar (4.37 against 4.58 Gauss) in both samples and range with values of radicals or free electrons (between 3-15 Gauss). The higher value of line width for $\mathrm{A}_{\mathrm{NW}-2}$ than for $A_{N W-1}$, suggest that interaction between dipoles in the solid would suffer a decrease in the magnetic local field due to an increase in the magnetic electrons detected.

This could be consequence of the formation of the superoxide paramagnetic $\mathrm{KO}_{2}$ during $\mathrm{KOH}$ decomposition in Saccharose carbonization process [7]. Concerning to SQUID analysis, two basic data sets were obtained for $\mathrm{A}_{\mathrm{NW}-1}$ and $A_{\mathrm{NW}-2}$. The first one described magnetization as a function of applied field at different temperatures, and the second data set described magnetization as a function of temperature at some constant applied field. Reproducibility of magnetic data was made by duplicate. Figs. $(3,4)$ show the relationship between magnetization and applied magnetic field at various temperatures for $A_{N W-1}$ and $A_{N W-2}$, respectively, in 


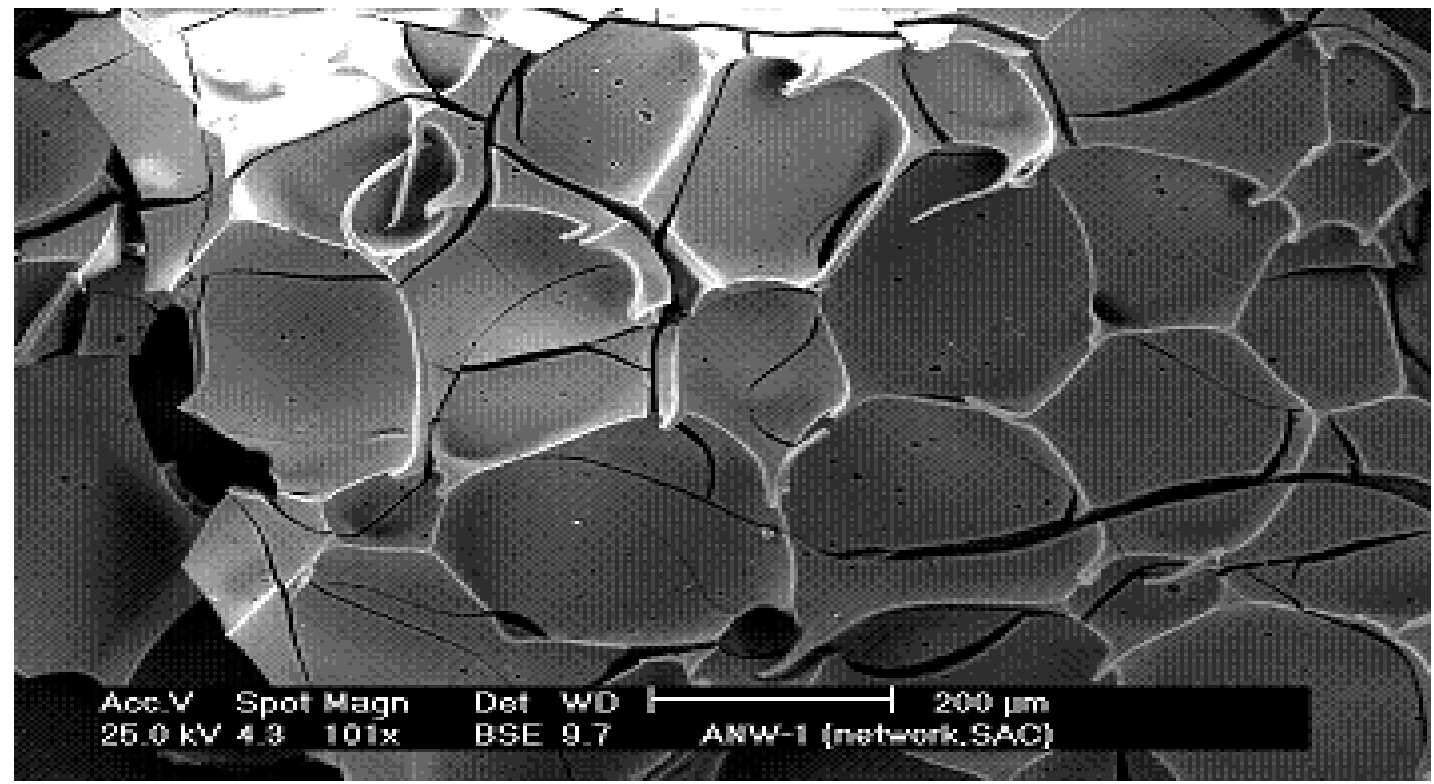

Fig. (1). SEM image of $A_{N W-1}$ (water dissolved ex-Saccharose [5]).

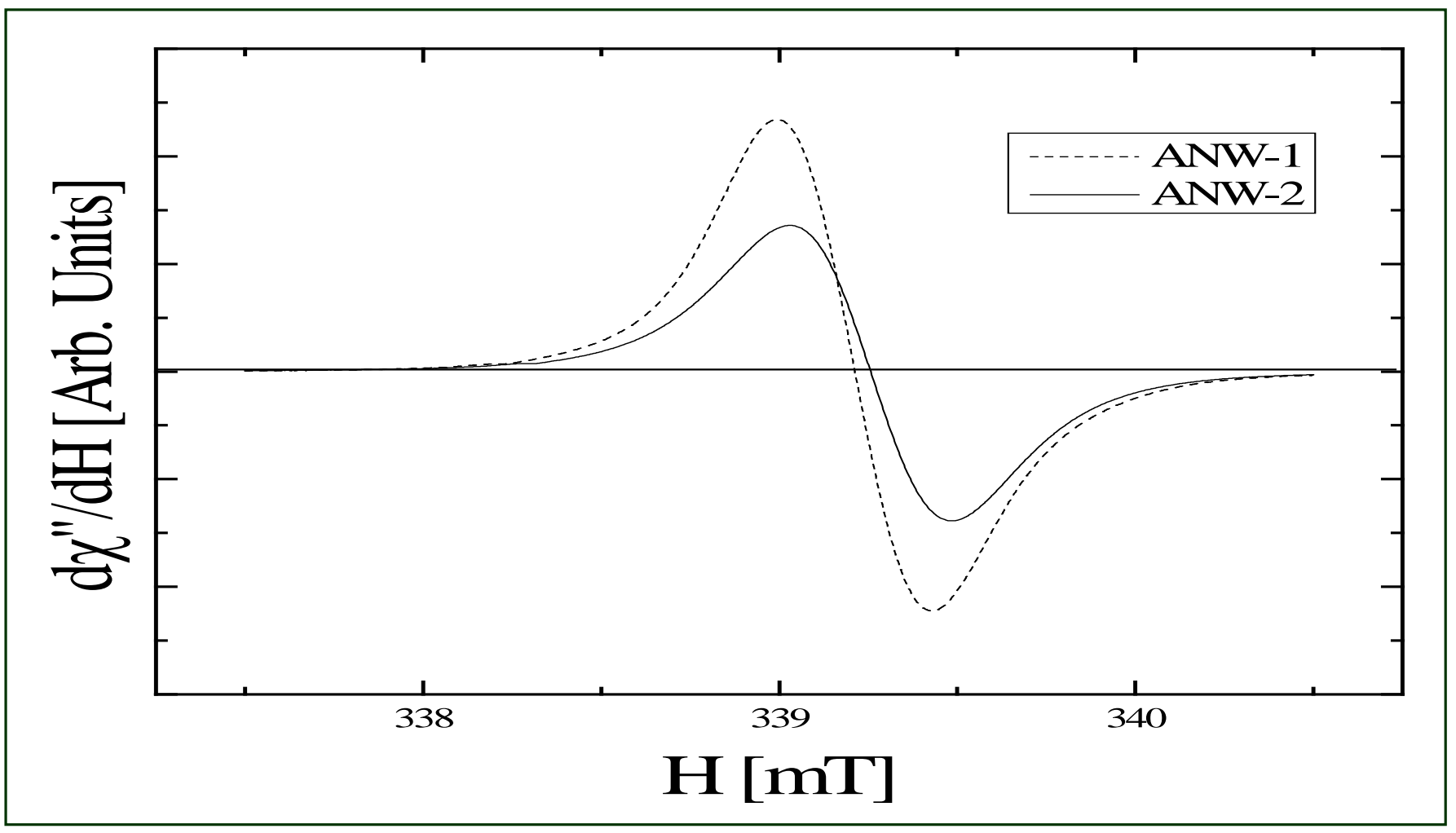

Fig. (2). EPR spectra of $A_{N W-1}$ and $A_{N W-2}(K O H$ aqueous solution dissolved ex-Saccharose [6]).

the temperature ranges from $1.8 \mathrm{~K}$ to $300 \mathrm{~K}$ and the field ranges from $0 \mathrm{~T}$ to $5 \mathrm{~T}$. As is readily observable from these plots, the magnetization of $\mathrm{A}_{\mathrm{NW}-1}$ and $\mathrm{A}_{\mathrm{NW}-2}$ is the result of paramagnetic and a diamagnetic component. At low temperatures, $1.8 \mathrm{~K}-10 \mathrm{~K}$, the paramagnetic component is significant in relation to the diamagnetic component, and thus both $\mathrm{A}_{\mathrm{NW}-1}$ and $\mathrm{A}_{\mathrm{NW}-2}$ are standard paramagnets at low tempera- ture. As the temperature is varied from $10 \mathrm{~K}$ to $300 \mathrm{~K}$, the diamagnetic component in both $\mathrm{A}_{\mathrm{NW}-1}$ and $\mathrm{A}_{\mathrm{NW}-2}$ outweighs the paramagnetic component, and what we observe is a standard diamagnetic compound.

Figs. $(5,6)$ illustrate the relationship between magnetism and temperature, plotted as $\chi$ per $\mathrm{g}$ as a function of $\mathrm{T}$ in 1 $\mathrm{kOe}$ for $\mathrm{A}_{\mathrm{NW}-1}$ and in $2 \mathrm{kOe}$ for $\mathrm{A}_{\mathrm{NW}-2}$. The inverse relation- 


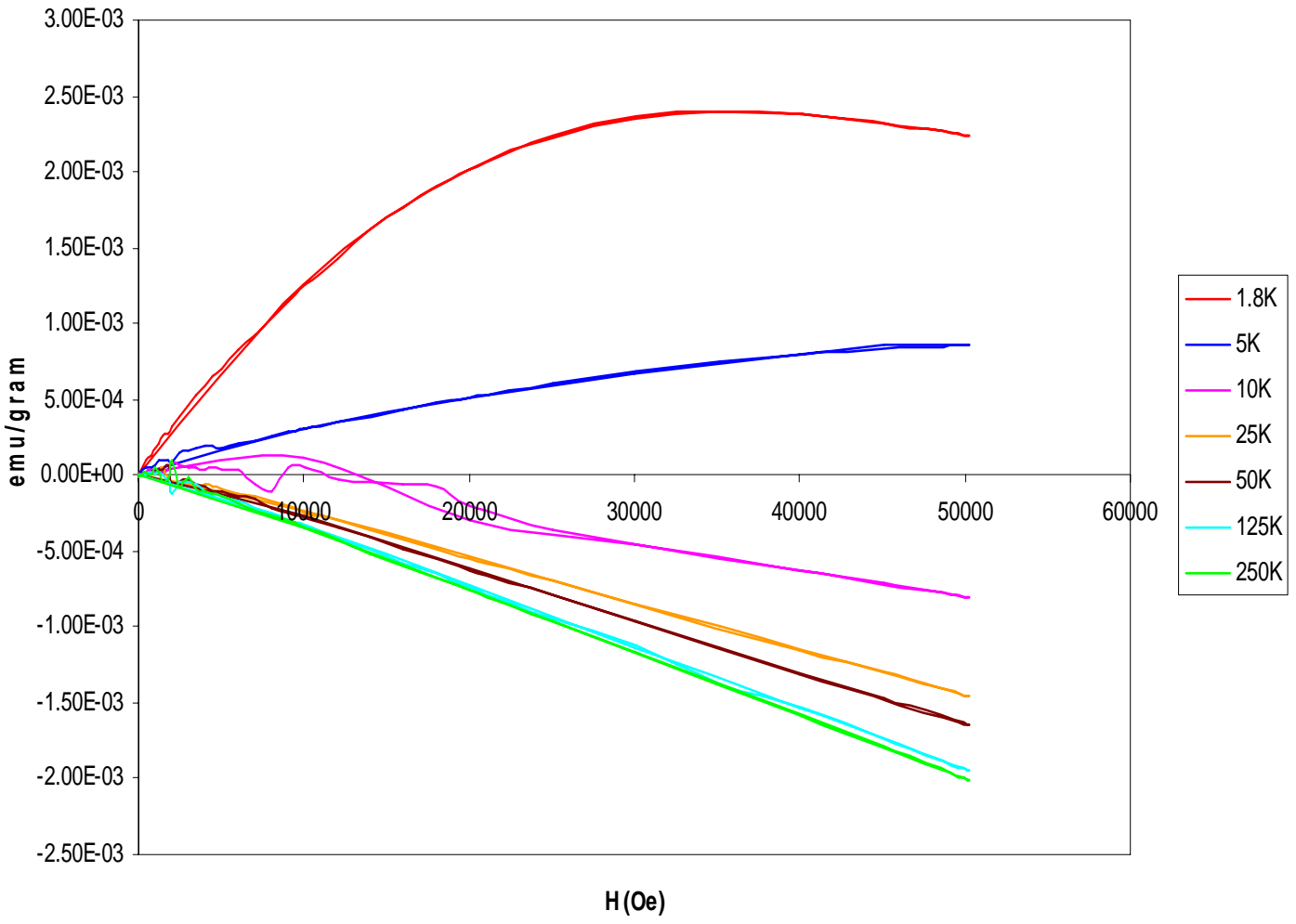

Fig. (3). Magnetization (M) as a function of $\mathrm{H}$ at various temperatures for $\mathrm{A}_{\mathrm{NW}-1}$

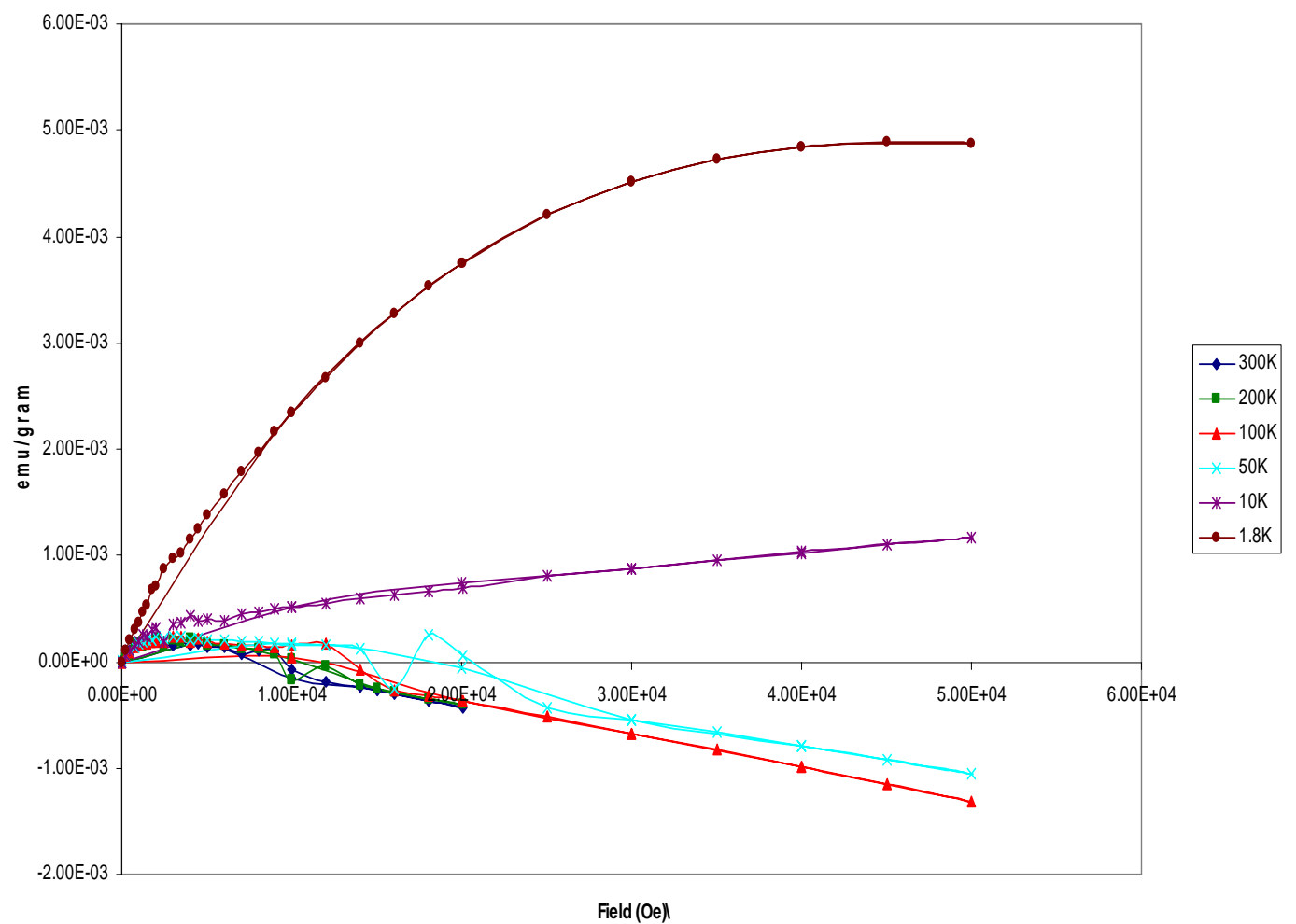

Fig. (4). Magnetization (M) as a function of $\mathrm{H}$ at various temperatures for $\mathrm{A}_{\mathrm{NW}-2}$

ship between $\chi$ and $\mathrm{T}$, which is indicative of a paramagnet, can be readily seen for both $\mathrm{A}_{\mathrm{NW}-1}$ and $\mathrm{A}_{\mathrm{NW}-2}$.

The data in Figs. $(\mathbf{5}, \mathbf{6})$ have been corrected for the intrinsic background of the sample holder, but no corrections have been made for either diamagnetizm, due to the variable na- ture of the structure and composition of the material. It must also be noted that the magnitude of the magnetization vector varies on the basis of the synthetic methods used to prepare the samples. This clearly reflects the variable number of free electrons or radicals in the samples based on the synthetic 


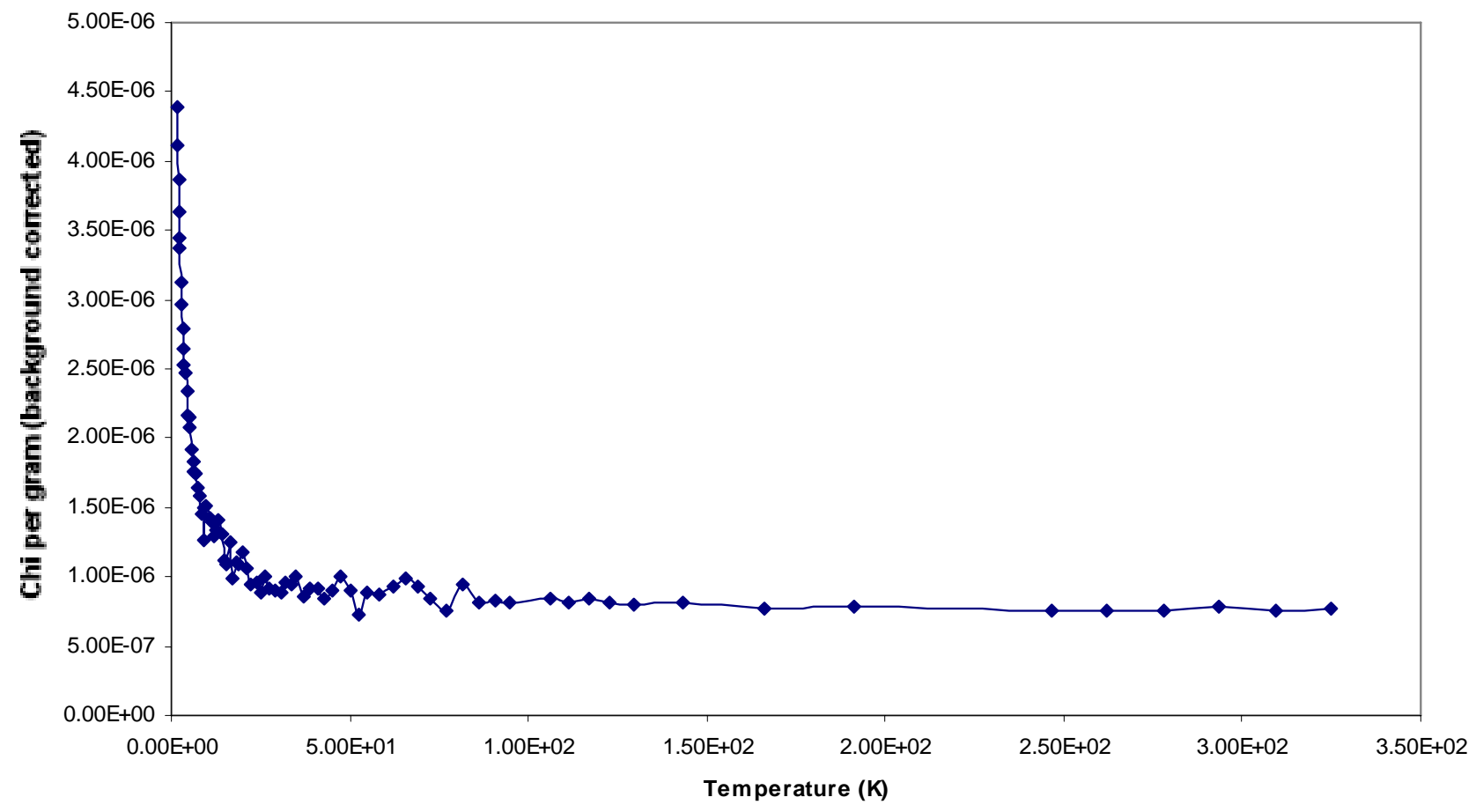

Fig. (5). $\chi / \mathrm{gm}$ as function of $\mathrm{T}(1 \mathrm{kOe})$ for $\mathrm{A}_{\mathrm{NW}-1}$.

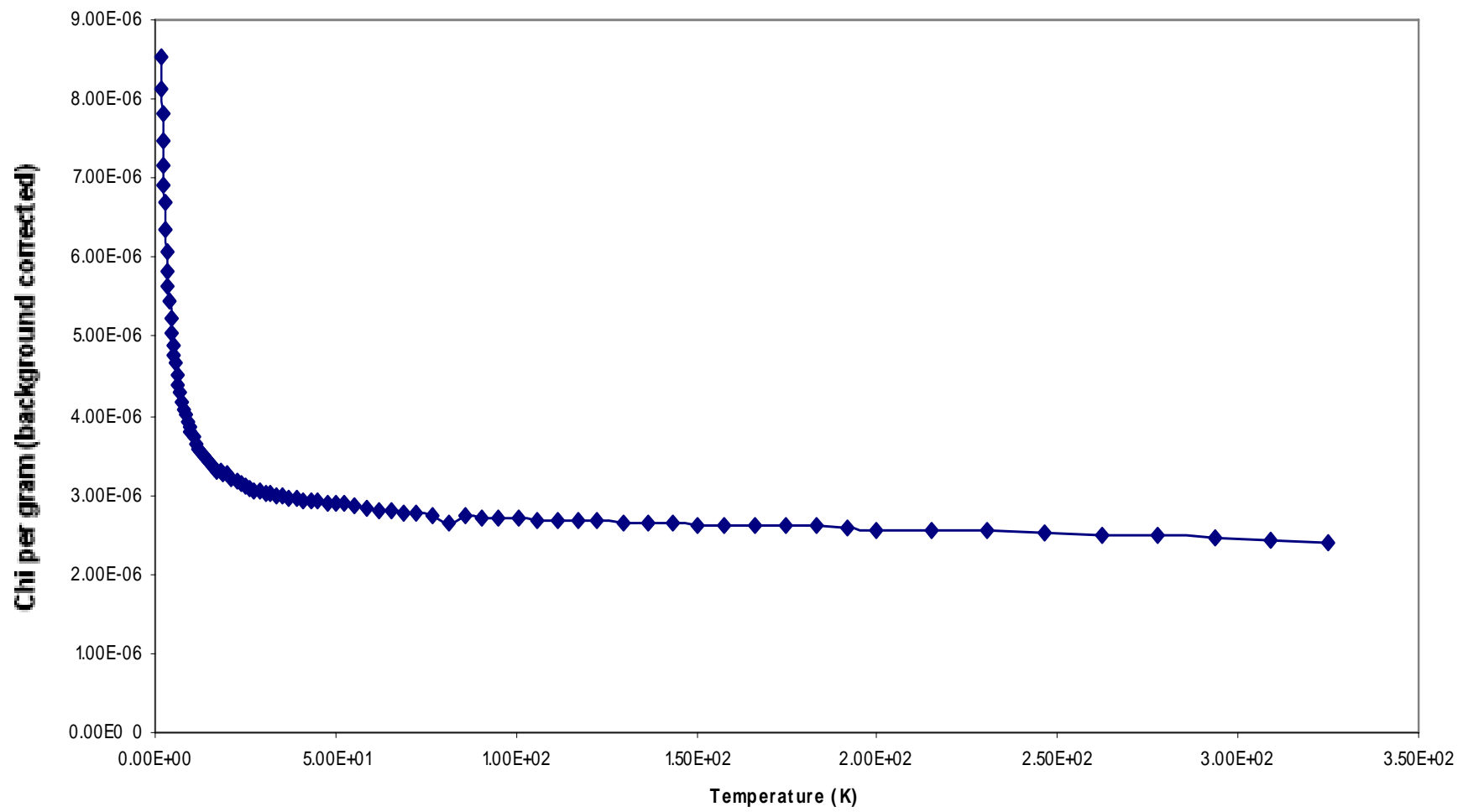

Fig. (6). $\chi / \mathrm{gm}$ as function of $\mathrm{T}(2 \mathrm{kOe})$ for $\mathrm{A}_{\mathrm{NW}-2}$.

approach utilized and network structure play an important role on the magnetic behavior of carbon foams. In conclusion, we confirmed that our samples were paramagnetic at low temperatures (i.e. $1.8 \mathrm{~K}-10 \mathrm{~K}$ ) and the diamagnetic temp- eratures are higher than $10 \mathrm{~K}$. Results showed that saccharose can be used as a precursor for the synthesis of carbon materials for magnetic applications at low temperature. 


\section{ACKNOWLEDGEMENTS}

J. Matos would like to thank Prof. Toshiaki Enoki and Prof. David Tomanek for the invaluable discussion with them during Carbon 2008 at Nagano.

\section{REFERENCES}

[1] Makarova TL. Magnetic properties of carbon structures. Semiconductors 2004; 38: 615-64.

[2] Harris PJF. Impact of the discovery of fullerenes on carbon science. Chem Phys Carbon 2003; 28: 1-39.
[3] Rode AV, Gamaly G, Christy AG, et al. Unconventional magnetism in all-carbon nanofoams. Phys Rev B 2004; 70: 0544071-9.

[4] Sato H, Kawatsu N, Enoki T, et al. Physisorption-induced change in the magnetism of microporous carbon. Carbon 2007; 45: 203-28.

[5] Matos J, Laine J. A carbon macro-network from the controlled pyrolisis of saccharose. J Mater Sci Lett 1998; 17: 649-51.

[6] Matos J, Labady M, Albornoz A, Laine J, Brito JL. Topological organization and textural changes of carbon macro-networks submitted to activation with $\mathrm{N}_{2}$ and $\mathrm{CO}_{2}$. J Mater Sci 2004; 39: 370516

[7] Matos J, Labady M, Albornoz A, Laine J, Brito JL. Catalytic effect of $\mathrm{KOH}$ on textural changes of carbon macro-networks by physical activation. J Molec Catal A: Chem 2005; 228: 189-94.

(C) Matos et al.; Licensee Bentham Open.

This is an open access article licensed under the terms of the Creative Commons Attribution Non-Commercial License (http://creativecommons.org/licenses/by$\mathrm{nc} / 3.0 /$ ) which permits unrestricted, non-commercial use, distribution and reproduction in any medium, provided the work is properly cited. 